\title{
ERRATUM: HIGHER ORDER AVERAGING THEORY FOR FINDING PERIODIC SOLUTIONS VIA BROUWER DEGREE
}

\author{
JAUME LLIBRE ${ }^{1}$, DOUGLAS D. NOVAES ${ }^{1,2}$ AND MARCO A. TEIXEIRA ${ }^{2}$
}

In the paper [1] instead of the matrices $e^{\eta(t, z)}, e^{-\eta(t, z)}$, and $M(z)$ it must be the matrices $Y(t, z), Y(t, z)^{-1}$, and $I d$ the identity matrix, respectively. The matrix $Y(t, z)$ is the $n \times n$ fundamental matrix solution of the periodic linear differential system

$$
u^{\prime}(t)=\partial F_{0}(t, \varphi(t, z)) u(t),
$$

such that $Y(0, z)=I d$ is the identity matrix, and $\varphi(t, z)$ a periodic solution of the unperturbed system $x^{\prime}(t)=F_{0}(t, x)$.

The change of these matrices appear in the pages $566,573,577$, and 578 .

\section{REFERENCES}

[1] J. Llibre, D.D. Novaes And M.A. TeixeIRA, Higher order averaging theorem for finding periodic solutions via Brouwer degree, Nonlinearity 27 (2014), 563 â 583 .

${ }^{1}$ Departament de Matematiques, Universitat Autònoma de Barcelona, 08193 Bellaterra, Barcelona, Catalonia, Spain

E-mail address: jllibre@mat.uab.cat, ddnovaes@mat.uab.cat

2 Departamento de Matematica, Universidade Estadual de Campinas, Caixa Postal 6065, 13083-859, Campinas, SP, Brazil

E-mail address: ddnovaes@ime.unicamp.br, teixeira@ime.unicamp.br 